Journal of Consumer Research, Inc.

The Devil Is in the Deliberation: Thinking Too Much Reduces Preference Consistency Author(s): Loran F. Nordgren and Ap Dijksterhuis

Reviewed work(s):

Source: Journal of Consumer Research, Vol. 36, No. 1 (June 2009), pp. 39-46

Published by: The University of Chicago Press

Stable URL: http://www.jstor.org/stable/10.1086/596306

Accessed: 11/07/2012 05:37

Your use of the JSTOR archive indicates your acceptance of the Terms \& Conditions of Use, available at

http://www.jstor.org/page/info/about/policies/terms.jsp

JSTOR is a not-for-profit service that helps scholars, researchers, and students discover, use, and build upon a wide range of content in a trusted digital archive. We use information technology and tools to increase productivity and facilitate new forms of scholarship. For more information about JSTOR, please contact support@jstor.org. 


\title{
The Devil Is in the Deliberation: Thinking Too Much Reduces Preference Consistency
}

\author{
LORAN F. NORDGREN \\ AP DIJKSTERHUIS*
}

\begin{abstract}
In five experiments we found that deliberation reduces preference consistency. In experiments 1 and 2, participants who deliberated on their preferences were less consistent in their evaluations compared to those who did not deliberate. Experiment 3 demonstrated that this effect is due to the impediment of deliberation and not to the benefit of nondeliberation. We hypothesized that deliberation leads to the inconsistent weighting of information, especially when the information is complex. As such, we predicted and found in experiments 4 and 5 that the extent to which deliberation decreases preference consistency depends upon the complexity of the information.
\end{abstract}

$\mathrm{C}$ onsumers expect and want consistent preferences. Preference consistency is important because consumer decisions, particularly significant consumer decisions, need to have enduring value. For example, selecting a new (and expensive) painting for the living room is done with the expectation that you will continue to find the painting beautiful for many years afterward. Even for goods that lose their value relatively quickly_-such as clothes and other fashion trends-people want their preferences to remain consistent throughout the expected time frame. A new bathing suit need not look good next season, but it should continue to look good throughout this season.

But how can we ensure that today's preferences will hold up tomorrow? The popular notion is that consistent preferences stem from a deliberative thought-processing strategy. We tend to trust an effortful process that reflects a careful, systematic consideration of all aspects of the evaluative object. Inconsistent evaluations, on the other hand, are believed to stem from a quick, intuitive thoughtprocessing strategy. Imagine the process of purchasing a car. The ostensibly best approach to making such an important decision would be to conduct a systematic analysis of each aspect of the car you are considering buying (e.g., poor safety rating, great gas mileage, and average durability, etc.) and then to determine the relative importance of each of

*Loran F. Nordgren is an assistant professor of management and organizations at the Kellogg School of Management, Northwestern University, Evanston, IL 60208 (L-Nordgren@kellogg.northwestern.edu). Ap Dijksterhuis is professor of psychology at Radboud University Nijmegen, Nijmegen, The Netherlands (a.dijksterhuis@psych.ru.nl).

John Deighton served as editor and Stephen Nowlis served as associate editor for this article.

Electronically published December 22, 2008 these features (e.g., with today's oil prices, gas mileage is the most important factor to consider). Basing one's judgment on this kind of careful, deliberative approach would seem to be the best platform for enduring preferences (Kahneman, Slovic, and Tversky 1982).

Yet, there is reason to believe that, rather than being beneficial, deliberation may in fact lead to more inconsistent preferences. This notion finds support in the growing body of research that demonstrates that conscious thought often undermines the quality of people's judgments (Dijksterhuis and Nordgren 2006).

Wilson and his colleagues, for example, have shown that introspective reasoning leads to a variety of suboptimal judgments. They have found that deliberation reduces satisfaction with personal judgments (Wilson et al. 1993) and attitude-behavior consistency (Wilson et al.1984) and leads to less agreement with expert opinion about an evaluative object (Halberstadt and Levine 1999; Johnson and Raab 2003; Wilson and Schooler 1991).

This counterintuitive finding has generally been thought to be a consequence of the limited processing capacity of deliberative thought (see Dijksterhuis [2004]; Dijksterhuis and Nordgren [2006]; Dijksterhuis et al. [2006] for more details). In this sense deliberation resembles a spotlight: deliberation focuses attention on a specific aspect of an object but is too narrow to take all aspects into account. This narrow focus disturbs the natural weighting of attributes by putting disproportionate weight on attributes that are accessible, plausible, and easy to verbalize and therefore puts too little weight on other attributes. According to Wilson and colleagues $(1993,332)$, "introspection . . . can change an optimal weighing scheme into a suboptimal one. When people analyze reasons, they might focus on those attributes 
of the attitude object that seem like plausible causes of the evaluations but were not weighted heavily before."

To demonstrate this point, Wilson and colleagues (1993; see also Dijksterhuis and van Olden 2006) had participants choose between art posters under two different conditions. Some participants were asked to analyze their thoughts by listing their reasons for liking or disliking each poster, whereas control participants merely made a choice. All participants were given their favorite art poster to take home and were phoned a few weeks later. Participants who analyzed their choices were less satisfied with their posters than control participants. Crucially, they demonstrated that deliberation hindered the evaluative process because it led people to focus on a limited number of attributes at the expense of other relevant attributes.

Although these studies demonstrate that deliberation can undermine the weighting of attributes, it is unclear whether suboptimal weighting undermines preference consistency. After all, the poor weighting of information can itself be consistent. If one judges the attractiveness of various apartments solely on the basis of the number of electrical sockets in the kitchen, one would see this as an instance of poor weighting. However, as long as one counts correctly, one will reach the same judgment each time. For deliberation to hinder consistency it requires that deliberation leads to inconsistent weighting on information.

Some evidence for this notion comes out of a study by Levine, Halberstadt, and Goldstone (1996). They had participants evaluate a large number of faces that varied along six dimensions (such as the shape of the nose). Participants either merely evaluated these faces or they had to think about the reasons for their evaluations before doing so. Using multidimensional scaling they found that participants who deliberated displayed less consistent weighting of the attributes from trial to trial. For example, a deliberator might at one point find a hooked nose to be attractive and later on find it less so. It is important to note that their study examined how consistently deliberators evaluated features of the face (i.e., aspects of the object) but did not examine whether deliberation led to more inconsistent evaluations of the object itself. This issue is taken up in the present study.

We hypothesized that deliberation reduces the consistency of consumer preferences. This hypothesis was based on the knowledge that deliberation leads to poor weighting (as is shown by evidence) and the idea that deliberation causes inconsistent weighting of attributes. Deliberation, one may say, introduces decisional noise by poor and unsystematic weighting.

\section{THE PRESENT STUDIES}

In five experiments we examined the prediction that deliberation reduces preference consistency. The same basic paradigm was used in each experiment. Participants were asked to evaluate a set of objects (between 7 and 12) at the beginning of the experimental session and then rate the same objects again 50 minutes later. Half of the participants made their evaluations deliberatively, and the other half made their evaluations without deliberation. In experiment 1 participants evaluated Chinese ideograms. In experiment 2 participants rated high- versus low-quality paintings, which enabled us to examine whether the consistency that nondeliberation affords comes at the cost of accuracy. With the aid of a control condition, experiment 3 tested whether the relative difference between the deliberation and nondeliberation conditions in the previous experiments is due to the benefits of a nondeliberative approach or to the limitation of a deliberative approach (as we predict).

Finally, experiments 4 and 5 examined how deliberation hinders consistency by testing a moderator that stems directly from our reasoning that deliberation disturbs the natural weighting of information. When the information that a preference is based on is very simple, weighting is hardly an issue (the deliberative spotlight captures all the information). Hence, we hypothesized that when the judgment task was very simple, the difference in inconsistency between deliberators and nondeliberators would disappear.

\section{EXPERIMENT 1}

This experiment examined the effect of deliberative, as compared to nondeliberative, evaluation on the consistency of people's preferences. We asked participants to rate a number of Chinese ideograms for attractiveness on two separate occasions. We predicted that deliberators would demonstrate more inconsistent preferences than nondeliberators.

\section{Methods}

Participants. Thirty-two undergraduate students (22 women and 10 men) participated for course credit or for money (7 euros).

Procedure. Participants were seated at a computer in a private cubicle. Participants were randomly assigned to one of two conditions, the deliberative or the nondeliberative. Participants were told, "you are about to see a number of Chinese ideograms. We would like you to rate how attractive you find each ideogram." Participants in the deliberation condition were then told, "New research has shown that people can make more accurate judgments about the attractiveness of objects if they make very deliberate decisions. This means that for each ideogram you should think very hard about the object, trying to generate clear reasons why you find it attractive or unattractive, before making your final evaluation." Participants in the nondeliberative condition were told, "New research has shown that people can make more accurate judgments about the attractiveness of objects if they make quick decisions based on a gut feeling. This means that for each ideogram you should base your judgment on your first impression of the ideogram. If the first moment you look at an ideogram it seems attractive (or unattractive), then rate it as such." The instructions for 
the second session closely followed the first session instructions.

Participants rated 12 ideograms at the beginning and end of the experimental session. There were approximately 50 minutes separating the two time sessions. Of the 12 ideograms presented at each session, eight identical ideograms were used in both sessions, and eight ideograms were used in only one of the sessions. Thus, each session contained the eight repeated ideograms and four unique ideograms. Our analyses focus only on those eight repeated ideograms. Adding the additional ideograms was done to mask the purpose of the study and to render explicit memory for individual ideograms unlikely.

In each session, the ideograms were presented in random order. Participants rated each ideogram on a 100-millimeter scale, with Very Unattractive and Very Attractive as the end points on the scale. Although there were no values visible on the scale (in order to make explicit memory for the evaluations impossible), scores ranged from -25 to 25 . For each ideogram we measured the evaluative rating and the time it took to make the evaluation. Consistency was measured as the absolute difference between the first and second evaluations, with scores ranging from zero (entirely consistent) to 50 (entirely inconsistent).

\section{Results}

Manipulation Check. We expected that participants who were asked to deliberate would take more time in making their evaluation than participants who were not asked to deliberate. To ensure that the manipulation was successful we compared the average amount of time each condition evaluated the ideograms. To calculate this comparison we averaged the eight decision times at each of the two sessions. As predicted, in the first session participants in the deliberative condition evaluated each ideogram for a longer period of time $(M=4.62$ seconds; $\mathrm{SD}=2.41)$ than participants in the nondeliberative condition $(M=2.40$ seconds; $\mathrm{SD}=0.75, F(1,32)=12.36, p=.001), \eta^{2}=.29$. Likewise, in the second session participants in the deliberative condition evaluated each ideogram for a longer period of time $(M=3.37$ seconds; $\mathrm{SD}=1.37)$ than participants in the nondeliberative condition $(M=1.88$ seconds; $\mathrm{SD}=$ $0.57, F(1,32)=16.11, p<.001), \eta^{2}=.35$.

Preference Consistency. We next examined the prediction that participants who made deliberative evaluations were more inconsistent in their preferences. For each ideogram we measured the absolute difference in rating from time 1 and time 2 and then averaged these eight absolute difference scores to generate one overall inconsistency score. In this and the remaining studies, higher scores characterize greater inconsistency. As predicted, participants who made deliberative evaluations were more inconsistent in their preferences $(M=7.85 ; \mathrm{SD}=2.93)$ than participants who made nondeliberative evaluations $(M=5.83$; $\mathrm{SD}=2.54, F(1,32)=4.30, p=.05), \eta^{2}=.13$.

\section{EXPERIMENT 2}

In the previous experiment we demonstrated that a deliberative approach can decrease the consistency of one's preferences. This finding provides initial evidence for the benefits of a nondeliberative processing style. One limitation of this conclusion might be that the consistency gained by a nondeliberative approach comes at the cost of some other aspect of the evaluation, such as the accuracy of the judgment. To address this issue, we asked participants to evaluate the quality of paintings. Half of these paintings are considered to be of the highest quality (they belong to the Museum of Modern Art in New York [MOMA]), and half are considered to be of the lowest quality (they belong to the Museum of Bad Art in Boston [MOBA]). This design enabled us to assess both the consistency and the accuracy of participant's preferences.

\section{Methods}

Participants. Seventy-three students (53 women and 20 men) participated for course credit or for money (7 euros).

Procedure. Participants were asked to rate eight paintings, four high-quality, MOMA paintings, and four lowquality, MOBA paintings (paintings were downloaded from the institutions' respective Web sites). As in the previous experiment, participants rated the paintings once at the beginning of the experimental session and again at the end of the experimental session (approximately 50 minutes later). Additional paintings were included at both time points in order to mask the purpose of the experiment. The instructions for the deliberators and nondeliberators were modified from those in experiment 1 .

\section{Results}

Manipulation Check. As in the previous experiment, at time 1 participants in the deliberation condition spent longer evaluating the paintings $(M=3.93$ seconds; $\mathrm{SD}=2.33)$ than participants in the nondeliberative condition $(M=2.30 \mathrm{sec}-$ onds; $\mathrm{SD}=1.15, F(1,73)=14.85, p<.001), \eta^{2}=.17$. Likewise, at time 2 participants in the deliberation condition spent longer evaluating the paintings $(M=2.26$ seconds; $\mathrm{SD}=1.08)$ than participants in the nondeliberative condition $(M=1.58$ seconds; $\mathrm{SD}=0.39, F(1,73)=13.21$, $p=.001), \eta^{2}=.15$.

Preference Consistency. We tested whether deliberation hindered the consistency of participants' preferences for the paintings. As expected, participants who deliberated were more inconsistent in their preferences $(M=3.82$; $\mathrm{SD}=$ 1.83) than participants who did not deliberate $(M=3.09$; $\mathrm{SD}=1.38, F(1,73)=3.81, p=.05), \eta^{2}=.05$. This result replicates the findings from experiment 1 .

Preference Accuracy. We next examined whether, collapsing across conditions, participants could distinguish be- 
tween the quality of the MOMA and MOBA paintings. Participants were able to differentiate between the two types of paintings, as they rated the MOMA paintings to be of higher quality $(M=-0.74)$ than the MOBA paintings $(M=-3.21 ; t(73)=3.01, p=.004)$. Yet crucially, deliberators did not rate MOMA (high-quality) paintings as more attractive $(M=-1.24$; SD $=6.06)$ than nondeliberators $(M=-0.27 ; \mathrm{SD}=6.81, t(73)=-.63$, NS $)$. Likewise, deliberators did not rate MOBA (low-quality) paintings as less attractive $(M=-2.80 ; \mathrm{SD}=7.12)$ than nondeliberators $(M=-3.60 ; \quad \mathrm{SD}=7.75, \quad t(73)=.46$, NS). In fact, to the extent there is any difference at all, the nondeliberators appear to be more accurate in their evaluations. Taken together, these findings suggest that not only does a nondeliberative approach lead to more consistent preferences, but it does so without sacrificing the accuracy of the judgment.

\section{EXPERIMENT 3}

The previous experiments have demonstrated a relative difference in preference consistency between the deliberation and nondeliberation conditions. Our hypothesis entails that deliberation disrupts consistency. But whereas we pressed people to deliberate in our experimental conditions, we also explicitly asked people not to deliberate in other conditions. For this reason, it is not fully clear what drives our effect. After all, some evidence suggests that people evaluate objects almost instantaneously (Giner-Sorolla, Garcia, and Bargh 1999). If evaluative judgments are by default automatic, the difference in consistency might be due to the advantage of the nondeliberative condition as opposed to the disadvantage of deliberation.

Thus, experiment 3 examined whether deliberation hinders preference consistency (as we propose) or whether nondeliberation enhances preference consistency, or both. To test this idea we compared a deliberation and nondeliberation condition with a no-instruction or control condition. Based on our hypothesis that deliberation causes inconsistent weighting of attributes, we expected that a deliberative processing strategy actively hinders preference consistency. In other words, we predicted that participants in the deliberation condition would form more inconsistent preferences than would participants in the nondeliberation and no-instruction conditions. Moreover, we expected no differences in preference consistency between the nondeliberation and the no-instruction conditions.

In this experiment we measured the consistency of taste preferences. We had participants rate their preferences for a variety of flavors such as chocolate, vanilla, and cinnamon. Experiments 1 and 2 involved forming visual-based preferences-participants looked at photos of ideograms or abstract art. In this experiment we had participants form tastebased preferences. Thus, this study also tested whether the inconsistency effects found in the earlier experiments generalize to different perceptual systems.

\section{Methods}

Participants. Sixty students (42 women and 18 men) participated for course credit or for money (5 euros).

Procedure. In this experiment participants were asked to indicate their preference for jellybean flavors. Participants evaluated 15 jellybeans at the beginning and end of an experimental session. There were approximately 40 minutes separating the two time sessions. Of the 15 jellybean flavors presented at each session, six were used in both sessions: chocolate, lemon, cinnamon, coffee, watermelon, and bubblegum. Our analyses focus only on the six repeated flavors, as adding the additional flavors was done to mask the purpose of the study and to render explicit memory for individual flavors unlikely.

In each session, participants were presented with 15 paper cups. Each cup was labeled with a number from one to 15. Participants were given a randomly generated number sequence and were instructed to taste each jellybean in the assigned order. Participants rated each jellybean on a 100millimeter scale, with Very Bad and Very Good as the end points on the scale (as in the previous experiments). The deliberation and nondeliberation conditions used instructions adapted from the previous experiments. Participants in the no-instruction condition were simply instructed to taste each jellybean in the assigned order.

\section{Results}

Manipulation Check. As predicted, at time 1 participants in the deliberation condition spent more time evaluating the flavors $(M=8.22$; SD $=2.23)$ than did participants in the nondeliberation condition $(M=5.70$; $\mathrm{SD}=$ 1.34) and, crucially, the no-instruction condition $(M=$ 6.17; $\mathrm{SD}=1.16, F(2,60)=13.30, p=.001), \eta^{2}=.32$. Likewise, at time 2 participants in the deliberation condition spent more time evaluating the flavors $(M=6.14$; $\mathrm{SD}=$ 1.52) than did participants in the nondeliberation condition $(M=4.46 ; \mathrm{SD}=1.27)$ and the no-instruction condition $(M$ $=4.98 ; \mathrm{SD}=0.89, F(2,60)=14.95, p=.001), \eta^{2}=.25$. At both time 1 and time 2, decision time did not differ between the nondeliberation and no-instruction conditions.

Preference Consistency. We predicted that participants in the deliberation condition would form more inconsistent preferences than would participants in the nondeliberation and no-instruction conditions. As predicted, the deliberation condition formed more inconsistent preferences $(M=$ 4.72; $\mathrm{SD}=1.66)$ than did the nondeliberation condition $(M=3.19 ; \mathrm{SD}=1.71)$ and the no-instruction condition $(M=3.44 ; \quad \mathrm{SD}=2.21, \quad F(2,60)=3.81, \quad p=.028)$, $\eta^{2}=.11$. Moreover, we found no difference in preference consistency for the nondeliberation and no-instruction conditions. This experiment supports our view that a deliberative processing style actively hinders preference consistency. 


\section{EXPERIMENT 4}

The previous experiments demonstrated that deliberation decreases the consistency of people's preferences. As in Wilson's poster study, we explain this finding in terms of deliberation's tendency to disturb the natural weighting of information. As such, we think that there are some circumstances in which deliberation may not hamper preference consistency. Specifically, we expect that the complexity of the evaluation can moderate this effect. Since inconsistency is a consequence of poor weighting, creating a very simple situation in which weighting hardly plays a role should make deliberate preferences more consistent.

In the present experiment we examined the moderating role of complexity on preference consistency in the context of judging six hypothetical apartments. Half of the apartments were very simple (contain three attributes) and half were complex (contain nine attributes). We expected deliberation to lead to greater inconsistency when evaluating the complex apartments, and we expected this effect to attenuate when evaluating the simple apartments.

\section{Methods}

Participants. Ninety students (66 women and 24 men) participated for course credit or for money (7 euros).

Procedure. Participants were told that they were going to be presented with information about six hypothetical apartments (labeled A-F). Each apartment was described by three primary attributes (location, price, and size). Half of the apartments were only described in terms of these three primary attributes (simple apartments). The other half of the apartments were described in terms of the three primary attributes and six additional secondary attributes, such as whether the apartment has carpet, a terrace, or a train stop nearby. The attributes were presented in random order. Each attribute was presented on the center of the screen for 2 seconds, followed immediately by the next attribute. After viewing the attributes, participants evaluated the apartment on the same continuous scale used in the previous experiments, from Very Negative to Very Positive. The apartments were presented in random order. Otherwise, instructions were identical to experiment 1 .

\section{Results}

Manipulation Check. The manipulation was successful. In the first session participants in the deliberation condition spent more time evaluating the apartments $(M=3.52 \mathrm{sec}-$ onds; $\mathrm{SD}=1.24)$ than participants in the nondeliberation condition $(M=2.75$ seconds; $\mathrm{SD}=1.02, \quad F(1,90)=$ $10.00, p=.002), \eta^{2}=.10$. Likewise, in the second session participants in the deliberation condition spent more time evaluating the apartments $(M=3.13$ seconds; SD $=1.09)$ than participants in the nondeliberation condition $(M=$ 2.51 seconds; $\mathrm{SD}=0.70, F(1,90)=9.80, p=.002)$, $\eta^{2}=.10$. Overall, there was also a difference in time spent evaluating the different types of apartment, such that participants spent more time evaluating complex apartments $(M=$ 3.37 seconds; $\mathrm{SD}=1.26)$ than simple apartments $(M=$ 2.65 seconds; $\mathrm{SD}=0.95, t(90)=4.88, p=.001)$.

Preference Consistency. We examined the effect of the manipulation on the preference consistency for the two apartment types (simple vs. complex) using a repeated measures ANOVA. There was a main effect for apartment type, as participants were more inconsistent in evaluating the complex apartments $(M=5.95 ; \mathrm{SD}=3.40)$ than the simple apartments $(M=4.70 ; S D=2.60, F(1,90)=6.00, p=$ $.01), \eta^{2}=.06$.

Crucially, there was an interaction between apartment complexity and processing style $(F=3.56, p=.06), \eta^{2}=04$. For the complex apartments, deliberation led to more inconsistent preferences $(M=6.60 ; \mathrm{SD}=3.27)$ than did nondeliberation $(M=5.18 ; \mathrm{SD}=3.13, F(1,90)=4.06, p=$ $.04), \eta^{2}=.04$. However, for the simple apartments there was no difference between the deliberation $(M=4.54$; $\mathrm{SD}=$ $2.75)$ and the nondeliberation conditions $(M=4.91$; $\mathrm{SD}=$ 2.43, $F(1,90)=0.46$, NS; see fig. 1$)$.

\section{EXPERIMENT 5}

The previous experiment provides evidence for the notion that complexity moderates the effect of deliberation on evaluative consistency. In that experiment complexity was defined objectively-the number of pieces of information each evaluative object contained. Yet, most objects we encounter do not offer such clearly defined dimensions. For instance, what makes an apartment simple or complex? If deliberation only hinders consistency for complex objects, the findings

\section{FIGURE 1}

MEAN INCONSISTENCY RATING OF ATTRACTIVENESS

(SIMPLE VS. COMPLEX APARTMENTS) BY CONDITION (DELIBERATIVE VS. NONDELIBERATIVE)

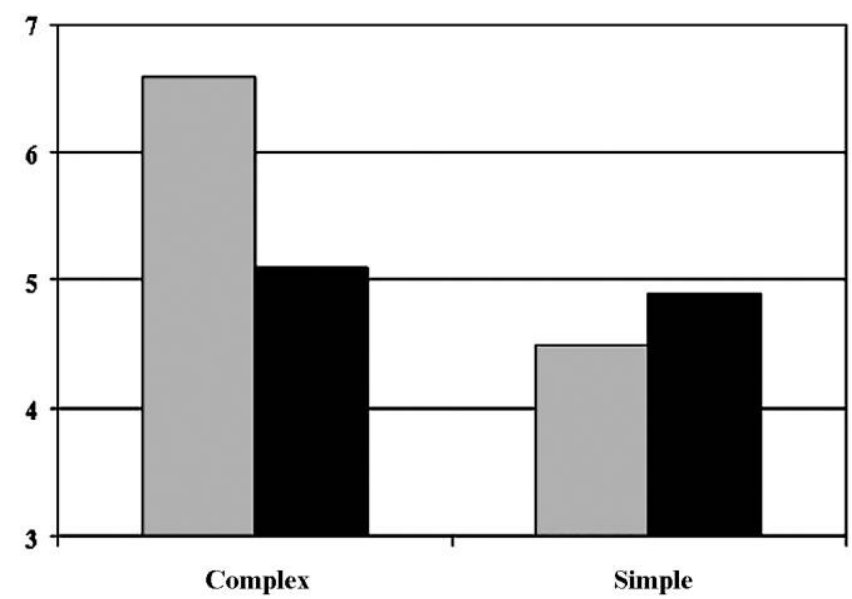

DDeliberation Non-deliberation 
from experiments 1 and 2 imply that the ideograms and painting used in those studies must have been complex. In what sense is an ideogram complex? In experiment 4 we argue for a "subjective complexity," reflected not in the characteristics of the object itself but, rather, determined by the richness of the evaluation.

We sought to replicate the findings from experiment 4 by demonstrating that the extent to which deliberation decreases preference consistency depends upon the complexity of the underlying weighting process. We manipulated the complexity of the weighting processes by pressing people to base their judgments on either one or several evaluative dimensions. We expected that the complex deliberators (based on more evaluative dimensions) would demonstrate greater inconsistency than would simple deliberators.

\section{Methods}

Participants. Ninety-three students (59 women and 34 men) participated for course credit or for money (7 euros).

Procedure. This experiment consisted of three conditions: simple deliberation, complex deliberation, and control deliberation. The procedure for the control deliberation condition was identical to the deliberation condition in experiment 1 . The aim of the simple deliberation condition was to have participants basis their attractiveness ratings only on one dimension of the ideogram-its symmetry: "When you see each ideogram carefully look at it and decide whether you think its form is symmetrical or asymmetrical and then rate it accordingly. For example, if you find symmetrical ideograms to be more attractive than asymmetrical ideograms, then rate a very symmetrical ideogram as very attractive."

In the complex deliberation condition participants were instructed to judge the ideograms in terms of five basic attributes: complexity, symmetry, line form (straight or rounded), dimension (vertical or horizontal), and composition (balanced or unbalanced). "When you see each ideogram carefully look at it and decide whether you think it is simple or complex, symmetrical or asymmetrical, etc., and decide whether these attributes create an attractive or unattractive ideogram." After these instructions, participants viewed sample ideograms to practice evaluating them in terms of the attributes that they were instructed to take into account. Otherwise, this experiment was identical to the deliberation condition in experiment 1 .

\section{Results}

Manipulation Check. In this experiment we measured the amount of time participants spent deliberating. Although there were no predictions about differences in decision time by condition, it seemed reasonable to assume that participants in the simple deliberation condition might spend less time deliberating than participants in the complex deliberation condition. There was in fact no significant difference for decision time among the three conditions. Nevertheless, decision time was used as a covariate in the subsequent analyses.

Preference Consistency. We predicted a linear trend, with participants who focused on only one attribute (simple deliberation) expected to be the most consistent in their preferences, whereas participants who incorporated a large number of attributes into their evaluation (complex deliberation) expected to be the least consistent. In support of this prediction, participants in the simple deliberation condition formed more consistent preferences $(M=5.61$; $\mathrm{SD}=2.69)$ than did participants in the control deliberation condition $(M=6.83 ; \mathrm{SD}=2.34)$ and the complex deliberation condition $(M=7.25 ; \mathrm{SD}=2.80, F(2,93)=3.53$, $p=.03$ ), $\eta^{2}=.07$ (see fig. 2).

This experiment replicates the earlier finding that complexity moderates the effect of deliberation on preference consistency and demonstrates that complexity does not need to be defined in terms of the objective features of a consumer object. Instead, it appears that subjective complexity-or the richness of the evaluation itself - can moderate deliberation's influence on preference consistency.

\section{GENERAL DISCUSSION}

People expect a degree of consistency from their consumer decisions. The present experiments examined the idea that how people think about consumer objects might influence the consistency of their preferences for those objects. Although we might expect consistent preferences to stem from careful, deliberative reasoning, we in fact found the very opposite. Whether evaluating abstract objects (Chinese

\section{FIGURE 2}

MEAN INCONSISTENCY RATING OF ATTRACTIVENESS BY DELIBERATIVE SCOPES (SIMPLE, CONTROL, OR COMPLEX)

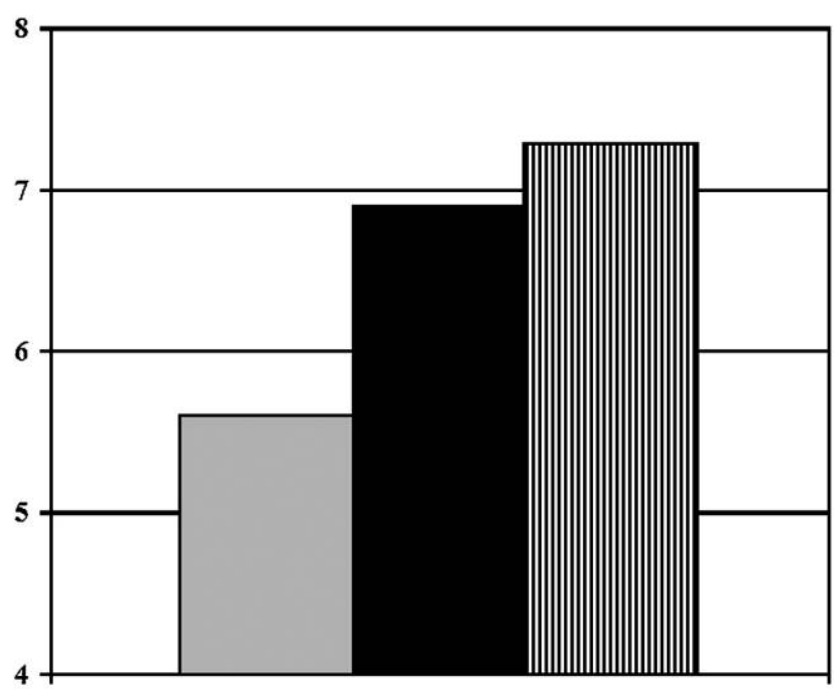

口Deliberation Simple @Deliberation Control @Deliberation Complex 
ideograms) or actual consumer items (paintings, apartments, and jellybeans), people who deliberated on their preferences were less consistent than those who made nondeliberative judgments.

The present research contributes to the growing literature on the problem of deliberating over consumer decisions. In the context of preference construction, for example, earlier work has shown that deliberation can hinder the systematic processing of consumer decisions (Toresdillas and Chaiken 1999). This work regards deliberation as a form of distraction, pulling attention away from the most relevant information. This perspective fits well with our finding that task complexity moderates the effect of deliberation on preference consistency. We found that the extent to which deliberation decreases preference consistency depends upon the complexity of the consumer object (experiments 4 and 5). We found that when the evaluative object (experiment 4) or the evaluative process (experiment 5) is complex, deliberation leads to inconsistent preferences. On the other hand, when the evaluative object or process is simple, deliberation is no longer a hindrance to preference consistency.

In our experiments, thinking more hindered consistency and did not improve decision accuracy (experiment 2). Our findings thus highlight the importance of nonconscious influences on consumer decision making (Bargh 2002; Dijksterhuis et al. 2005; Johar, Maheswaran, and Peracchio 2006). For example, nonconscious exposure to consumer information can have a substantial impact on consumer decisions (Shapiro, MacInnis, and Heckler 1997). Mandel and Johnson (2002), for example, found that priming product attributes through background colors and images influenced consumer choice, even though the participants had no insight into this effect. Likewise, research on the "deliberation-without-attention" effect has found, both in the laboratory as well as among actual shoppers, that purchases of complex products were more favorable when decisions were made in the absence of attentive deliberation (Dijksterhuis et al. 2006).

Although the take-home message of this article is that complex consumer decisions benefit from a nondeliberative evaluative process, the consequences of deliberation may have equally important implications for the marketplace. Earlier we compared deliberation to a spotlight-focusing attention on specific aspects of an object while ignoring other attributes. If deliberative attention naturally gravitates toward highly salient or novel aspects of an object, marketers might use a deliberative mind-set to focus consumers' attention toward particular attributes. For example, if a car boasts one particularly good feature (e.g., safety) but has a number of other negative features (e.g., expensive, bad gas mileage, poor handling), a car salesman might encourage a potential car buyer to deliberate over the pros and cons of the car, while at the same time emphasizing the importance of safety. In this way, the disturbed weighting of attributes created by deliberation might be used to highlight the one sellable feature and draw attention away from the unattractive features.
Although this is one of the few studies to address explicitly the issue of preference consistency, a host of psychological theories are at least in part grounded in the notion that consistency is a beneficial feature of judgments. The impact bias, for example, demonstrates that people often believe an emotional event will have a longer-reaching impact than it in fact does (Gilbert et al. 1998). One could argue that one reason for such biases is that people judge these events inconsistently. That is, what appears to be good now may appear to be less good later on, simply because our judgmental apparatus is far from flawless. And ironically, it gets worse when we deliberate.

\section{REFERENCES}

Bargh, John A. (2002), "Losing Consciousness: Automatic Influences on Consumer Judgment, Motivation, and Behavior," Journal of Consumer Research, 29 (2), 280-85.

$\rightarrow$ Dijksterhuis, Ap (2004), "Think Different: The Merits of Unconscious Thought in Preference Development and Decision Making," Journal of Personality and Social Psychology, 87 (5), 586-98.

$\rightarrow$ Dijksterhuis, Ap, Maarten Bos, Loran F. Nordgren, and Rick B. van Baaren (2006), "On Making the Right Choice: The Deliberation-without-Attention Effect," Science, 311 (5763), 1005-7.

$\rightarrow$ Dijksterhuis, Ap and Loran F. Nordgren (2006), "A Theory of Unconscious Thought," Perspectives on Psychological Science, 1 (2), 95-109.

$\rightarrow$ Dijksterhuis, Ap, Pamela K. Smith, Rick B. van Baaren, and Daniël Wigboldus (2005), "The Unconscious Consumer: Effects of Environment on Consumer Choice," Journal of Consumer Psychology, 15 (3), 193-202.

$\rightarrow$ Dijksterhuis, Ap and Zeger van Olden (2006), "To Think or Not to Think, or to Think Unconsciously Perhaps? Unconscious Thought Increases Post-choice Satisfaction," Journal of Experimental Social Psychology, 42 (5), 627-31.

$\rightarrow$ Gilbert, Daniel T., Elizabeth C. Pinel, Tim D. Wilson, Stephen J. Blumberg, and Thalia Wheatley (1998), "Immune Neglect: A Source of Durability Bias in Affective Forecasting," Journal of Personality and Social Psychology, 75 (3), 617-38.

$\rightarrow$ Giner-Sorolla, Roger, M. Garcia, and John A. Bargh (1999), "The Automatic Evaluation of Pictures," Social Cognition, 17(1), 76-96.

$\rightarrow$ Halberstadt, Jamin B. and Gary M. Levine (1999), "Effects of Reasons Analysis on the Accuracy of Predicting Basketball Games," Journal of Applied Social Psychology, 29 (3), 517-30.

Johar, Gita V., Durairaj Maheswaran, and Laura Peracchio (2006), "MAPping the Frontiers: Theoretical Advances in Consumer Research on Memory, Affect, and Persuasion," Journal of Consumer Research, 33 (1), 139-49.

$\rightarrow$ Johnson, Joseph G. and Marjus Raab (2003), "Take the First: Option-Generation and Resulting Choices," Organizational Behavior and Human Decision Processes, 91 (2), 215-29.

Kahneman, Daniel, Paul Slovic, and Amos Tversky (1982), Judgment under Uncertainty: Heuristics and Biases, Cambridge: Cambridge University Press.

$\rightarrow$ Levine, Gary M., Jamin B. Halberstadt, and Robert L. Goldstone (1996), "Reasoning and the Weighing of Attributes in Attitude Judgments," Journal of Personality and Social Psychology, 70 (2), 230-40. 
$\rightarrow$ Mandel, Naomi and Eric J. Johnson (2002), "When Web Pages Influence Choice: Effects of Visual Primes on Experts and Novices," Journal of Consumer Research, 29 (2), 235-45.

$\rightarrow$ Shapiro, Stewart D., Deborah J. MacInnis, and Susan E. Heckler (1997), "The Effects of Incidental Ad Exposure on the Formation of Consideration Sets," Journal of Consumer Research, 24 (2), 94-104.

$\rightarrow$ Tordesillas, Rosalind S. and Shelly Chaiken (1999), “Thinking Too Much or Too Little? The Effects of Introspection on the Decision-Making Process," Personality and Social Psychology Bulletin, 25 (5), 625-31.

$\rightarrow$ Wilson, Timothy D., Dana S. Dunn, Jane A. Bybee, D. B. Hyman, and J. A. Rotondo (1984), "Effects of Analyzing Reasons on Attitude-Behavior Consistency," Journal of Personality and Social Psychology, 47 (1), 5-16.

$\rightarrow$ Wilson, Timothy D., Douglas Lisle, Jonathan W. Schooler, Sara D. Hodges, Kristen J. Klaaren, and Suzanne J. LaFleur (1993), "Introspecting about Reasons Can Reduce Post-choice Satisfaction," Personality and Social Psychology Bulletin, 19 (3), 331-39.

$\rightarrow$ Wilson, Timothy D. and Jonathan W. Schooler (1991). “Thinking Too Much: Introspection Can Reduce the Quality of Preferences and Decisions," Journal of Personality and Social Psychology, 60 (2), 181-92. 